\title{
Identificação dos sinais neurocomportamentais de bebês pré-termo por profissionais que atuam na Unidade de Terapia Intensiva Neonatal (UTIN)
}

\author{
Identification of the preterm neurobehavioral \\ signs by the staff in Neonatal Intensive Care Unit \\ (NICU)
}

\author{
Lucieny Almohalha1, Ruth Maria Ribeiro Guerra ${ }^{2}$
}

ALMOHALLA, L., GUERRA, R. M. R. Identificação dos sinais neurocomportamentais de bebês pré-termo por profissionais que atuam na Unidade de Terapia Intensiva Neonatal (UTIN). Rev. Ter. Ocup. Univ. São Paulo, v. 22, n. 2, p. 117-126, maio/ago. 2011.

RESUMO: Introdução: Em resposta a estímulos externos comuns ao ambiente da UTIN, o recém nascido pré-termo (RNPT) emite sinais neurocomportamentais de retraimento ou de aproximação. Esses sinais oferecem dicas aos profissionais para suas intervenções. A modulação correta dos estímulos leva a um melhor desenvolvimento global do RNPT e os profissionais devem estar aptos para perceber e avaliar esses sinais. Objetivo: Investigar o nível de identificação, por parte dos profissionais da UTIN, dos sinais neurocomportamentais de retraimento e aproximação apresentados por RNPT e analisar os fatores que podem influenciar a aquisição de conhecimentos acerca desses sinais. Procedimentos metodológicos: Os dados foram coletados através da aplicação do Checklist da Assessment of Preterm Infants'Behavior (ALS \& cols., 1982) aos profissionais de uma UTIN e através da análise das respostas das questões abertas incluídas no instrumento. Os dados quantitativos foram analisados por contagem de freqüência e os dados qualitativos por análise de conteúdo. Resultados: Responderam ao checklist 45 profissionais, $80 \%$ de nível técnico e $20 \%$ de nível superior. Dos 16 sinais de retraimento e 17 sinais de aproximação possíveis de assinalar, os profissionais de nível superior observaram 03 sinais de retraimento e 07 sinais de aproximação a mais que os de nível técnico. Pela análise qualitativa, verificou-se que a correta identificação dos sinais do RNPT é influenciada por fatores como: extensiva jornada de trabalho, sutileza dos sinais apresentados pelos bebês, experiência profissional e a falta de capacitação.

DESCRITORES: Humanização da assistência; Manifestações neurocomportamentais; Prematuro; Pessoal de saúde.

\footnotetext{
* Trabalho apresentado na forma oral à I Mostra Científica de Terapia Ocupacional da UFTM, na cidade de Uberaba (MG) em 24 de junho de 2010 e na forma de póster no II Congresso Internacional de Saúde da Criança e do Adolescente, na cidade de São Paulo (SP) em 26 de agosto de 2010.

1. Graduada em Terapia Ocupacional pela Universidade Federal do Triângulo Mineiro (UFTM).

2. Terapeuta Ocupacional, Pós-graduanda do Programa de Residência Multiprofissional em Saúde - Atenção Integral a Saúde/ FMRP-USP. Endereço para correspondência: Prof. Lucieny Almohalha - Curso de Terapia Ocupacional da Universidade Federal do Triângulo Mineiro (UFTM). Rua Getúlio Guaritá, S/N, Bairro Abadia, CEP 38022-440, Uberaba, MG. e-mail: almohalha@to.uftm.edu.br; almohalha@gmail. com; ruthrguerra@gmail.com
} 


\section{INTRODUÇÃO}

Organização Mundial de Saúde (OMS)
define o recém-nascido pré-termo (RNPT)
como aquele que nasce antes da $37^{\mathrm{a}}$ semana de idade gestacional (IG). No século XX os avanços tecnológicos e científicos proporcionaram um grande avanço da medicina obstétrica e perinatal e das UTIN's ocorrendo aumento das taxas de sobrevida de recém-nascidos cada vez mais imaturos e de baixo peso ao nascimento. Muitas vezes esses RNPT necessitam de internação prolongada em condições de terapia intensiva (BÜHLER, 2008; COSTA; MARBA, 2006; GABRIEL, 2008; SCOCHI et al., 2001).

Devido a ênfase nos cuidados biológicos que permeia as intervenções na UTIN, há uma sobrecarga sensorial aos RNPTs, pois estes são expostos a estímulos externos pouco apropriados para sua maturação biológica, porém essenciais para a sua sobrevida. A imaturidade das funções orgânicas e anatômicas apresentadas pelo RNPT, principalmente de seu sistema nervoso, irão dificultar o processamento desses estímulos em nível central e inviabilizar respostas sensoriais e motoras adaptativas efetivas. Assim, o prognóstico de desenvolvimento do RNPT vai depender de sua capacidade de processamento neurológico e a complexa interação de fatores ambientais, sociais e biológicos (ALS, 2009; ANDREA; RODOLFO, 2006; CARVALHO et al., 2005; COSTA; MARBA, 2006; REICHERT et al., 2007; TRONCHIN; TSUNECHIRO, 2007).

As respostas comportamentais dos bebês prematuros a diversas situações que geram desconforto ou prazer, podem se mostrar aparentemente inespecíficas durante as intervenções clínicas. Assim, muitos profissionais de saúde em sua desgastante rotina de trabalho, consciente ou inconscientemente, podem não reconhecer ou banalizar estas respostas ou ainda se mostrarem indiferentes a elas (LAMY et al., 1997; SANTOS, 2007). Esta observação se torna necessária a fim de que os profissionais respeitem as individualidades de cada bebê durante sua atuação clínica, promovam o desenvolvimento saudável destes e ofereça uma intervenção integral, de qualidade e humanizada (REICHERT et al., 2007; SCOCHI et al., 2001).

A humanização deve ser vista como um direito do paciente em todo seu ciclo vital. Em uma visão humanizada de atendimento, todos têm direito ao respeito das suas necessidades, valores e crenças, princípios éticos e morais; ter alívio da dor e de seu sofrimento com todos os recursos tecnológicos e psicológicos disponíveis; e ainda, ter condições e ambientes que facilitem o restabelecimento, a manutenção, a melhora da saúde e, em última instância, a morte digna (MARTINS; FARIA, 2000). Segundo o Ministério da Saúde (2008) o conceito de humanização no campo da saúde é assumido por intermédio da Política Nacional de Humanização (PNH), que enfatiza a necessidade de assegurar atenção integral à população e estratégias para ampliar a condição de direitos e de cidadania das pessoas.

Muitas teorias relacionadas ao acolhimento do RNPT vão de encontro ao proposto pelo paradigma da PNH. Dentre estas se destaca a Teoria do Desenvolvimento Síncronoativo proposto por Heidelise Als (1986) que enfatiza o acolhimento mais humanizado aos recém-nascidos e delineia caminhos para a observação do funcionamento cerebral do bebê, via observação de seu comportamento. Esta teoria identifica o limiar do bebê em relação ao estresse, sua capacidade de auto-regulação e sua "real" disponibilidade de energia para procedimentos clínicos de acordo com o funcionamento dos subsistemas: autônomo, motor, de organização dos estados, de atenção e interação e sistema regulador. Esses subsistemas são interligados e interativos e promovem o estado de equilíbrio/homeostase do bebê. Devido a esta interdependência, pode-se observar que se existe uma desorganização de um determinado subsistema isso acarretará na sobrecarga dos demais e que será manifestada pelo bebê como alterações dos seus comportamentos (BRASIL, 2009; KUDO et al., 1997; MONTEIRO, 2007; PÊGO, 2007).

Segundo a abordagem síncrono-ativa, as alterações comportamentais dos RNPT em resposta aos estímulos advindos do meio são observáveis, sendo nomeadas como sinais de aproximação ou retraimento (ALS, 2009; BRASIL, 2009; KUDO et al., 1997). Os sinais de aproximação indicarão se o bebê está recebendo uma quantidade de estimulação e interação adequada convidando o profissional a intervir com ele. Os sinais de retraimento indicam que o bebê está muito estressado e as estimulações excessivas devem ser gradativamente retiradas indicando ao profissional a necessidade de esperar um momento mais adequado para realizar a intervenção. Assim, destaca-se a importância do profissional da UTI conhecer, identificar e intervir junto aos sinais neurocomportamentais do RNPT. Isto possibilitará um atendimento humanizado, com respeito ao desenvolvimento e ritmo de cada bebê, os quais estarão mais receptivos para os procedimentos clínicos (ALS, 2009; BRASIL, 2009).

Dentre as avaliações que abordam os sinais neurocomportamentais do recém-nascido, destaca-se a Assessment of Preterm Infants Behavior (APIB) (Als et 
al., 1982), sendo esta apropriada para bebês pré-termos, a termo e em risco de atrasos no desenvolvimento. Esta foca a avaliação das interações dos subsistemas com o ambiente, que são expressas pelo comportamento do bebê. A APIB é considerada clinicamente relevante aos profissionais para proporcionar uma intervenção individualizada adequada (Als et al., 1982).

A partir dos dados apontados pela literatura, observase que a articulação da atuação profissional em conjunto ao reconhecimento dos sinais neurocomportamentais do bebê é uma prática pouco difundida em várias UTIN's no país. Além disso, destaca-se a importância da identificação da linguagem do RNPT visando um atendimento de acordo com as necessidades de cada criança e, conseqüentemente, uma assistência mais humanizada. Assim, este estudo teve por objetivo investigar o nível de identificação, por parte dos profissionais da UTIN de um HC, dos sinais neurocomportamentais de aproximação e retraimento apresentados por RNPT; e analisar os fatores que podem influenciar a aquisição de conhecimentos acerca desses sinais.

\section{PROCEDIMENTOS METODOLÓGICOS}

Este estudo se caracterizou como uma pesquisa exploratória e foi realizado com profissionais que atuam em uma UTIN de um Hospital de Clínicas. Esta UTIN possui 20 leitos destinados a prematuros até crianças de 12 anos. A equipe foi constituída por 90 profissionais sendo: 44 técnicos de enfermagem, 15 auxiliares de enfermagem, oito enfermeiros, cinco escriturários, quatro fisioterapeutas, 11 médicos, uma fonoaudióloga, um assistente social e uma psicóloga. Foram incluídos na pesquisa todos os profissionais que atuam diretamente com o bebê. Os critérios de exclusão foram para os profissionais que estivessem de férias ou com licença saúde no período de coleta de dados.

Para a coleta de dados foi aplicado um checklist (anexo I) adaptado pelas autoras retirado de parte da APIB (ALS et al., 1982). Este checklist aborda os comportamentos de auto-regulação do bebê prematuro até o primeiro mês de vida. Os profissionais da UTIN assinalaram os sinais de aproximação e retraimento presentes no instrumento e que foram observados durante suas práticas.

No cabeçalho do checklist foram incluídas questões sobre a formação do profissional, o tempo de formado, o tempo de atuação na área e o tempo de atuação na unidade neonatal da pesquisa. Ao final do checklist, foram incluídas três perguntas que se relacionavam a dificuldades vivenciadas pelos profissionais para identificação dos sinais apresentados pelo bebê, conhecimento prévio destes sinais e observações que julgou necessário acrescentar.

A coleta de dados ocorreu no período de 11/12/2009 a 11/02/2010. Os checklists foram entregues na UTIN erecolhidos no dia ou no plantão seguinte do profissional. Os dados quantitativos foram analisados por análise estatística de freqüência relativa e absoluta e os dados qualitativos pela técnica de análise de conteúdo proposta por Bardin, em 1979.

A presente pesquisa foi submetida e aprovada em 10 de julho de 2009 pelo Comitê de Ética e Pesquisa da Universidade Federal do Triângulo Mineiro, protocolo 1414, respeitando a resolução 196/96. Os dados foram coletados depois de aceite dos profissionais pela assinatura do Termo de Consentimento Livre e Esclarecido.

\section{RESULTADOS}

Os resultados serão apresentados com a análise dos dados de todos os profissionais da UTIN que participaram da pesquisa e depois divididos por categorias de nível técnico (NT) e nível superior (NS). O primeiro nível corresponde aos técnicos e auxiliares de enfermagem, e o segundo aos enfermeiros, fisioterapeutas, fonoaudióloga, médicos e psicóloga.

Do total de 90 funcionários da UTIN 15 foram excluídos do estudo. Dos 75 profissionais incluídos e que foram entregues os checklists, $60 \%$ (45 profissionais) responderam ao checklist e retornaram o formulário ao pesquisador, sendo $80 \%$ deste do NT e $20 \%$ do NS. Do NT 53 profissionais foram incluídos no estudo e 36 (68\%) destes responderam ao checklist. Na categoria do NS 22 profissionais foram incluídos tendo respondido ao checklist 9 (41\%) destes. Houve uma menor adesão dos enfermeiros, seguido pelos médicos e total adesão das outras classes.

No cabeçalho do instrumento vinte e sete profissionais do NT e nove do NS responderam. Conforme observado na Tabela 1 houve predomínio do tempo de 1 a 5 anos, nos itens do cabeçalho, para todos os profissionais da UTI e ambas as categorias.

Os resultados relativos à contagem de freqüência dos sinais de aproximação e retraimento assinalados pelos profissionais da UTIN estão apresentados na Tabela 2. As colunas 2 e 6 correspondem à porcentagem dos sinais assinalados por toda a população pesquisada, as colunas $3 \mathrm{e}$ 7 correspondem à porcentagem assinalada pela população integrante do NT e as colunas 4 e 8 são relativas à porcentagem assinalada pela população integrante do NS. 
ALMOHAllA, L., GUERRA, R. M. R. Identificação. Rev. Ter. Ocup. Univ. São Paulo, v. 22, n. 2, p. 117-126, maio/ago. 2011.

Tabela 1. Tempo de formação, atuação na área de UTIN e na unidade pesquisada

\begin{tabular}{|c|c|c|c|c|c|c|c|c|c|}
\hline \multicolumn{4}{|c|}{ Profissionais UTIN } & \multicolumn{3}{|c|}{ Nível Técnico } & \multicolumn{3}{|c|}{ Nível Superior } \\
\hline Anos & $\begin{array}{l}\text { Tempo } \\
\text { de for- } \\
\text { mado }\end{array}$ & $\begin{array}{c}\text { Tempo de } \\
\text { atuação na } \\
\text { área }\end{array}$ & $\begin{array}{c}\text { Tempo de } \\
\text { atuação na } \\
\text { UTIN pesq. }\end{array}$ & $\begin{array}{l}\text { Tempo } \\
\text { de for- } \\
\text { mado }\end{array}$ & $\begin{array}{l}\text { Tempo de } \\
\text { atuação } \\
\text { na área }\end{array}$ & $\begin{array}{c}\text { Tempo de } \\
\text { atuação na } \\
\text { UTIN pesq. }\end{array}$ & $\begin{array}{c}\text { Tempo } \\
\text { de for- } \\
\text { mado }\end{array}$ & $\begin{array}{l}\text { Tempo de } \\
\text { atuação } \\
\text { na área }\end{array}$ & $\begin{array}{c}\text { Tempo de } \\
\text { atuação na } \\
\text { UTIN pesq. }\end{array}$ \\
\hline até 1a & $3 \%$ & $5 \%$ & $11 \%$ & $4 \%$ & $4 \%$ & $4 \%$ & 0 & $11 \%$ & $33 \%$ \\
\hline $1-5 a$ & $53 \%$ & $53 \%$ & $59 \%$ & $45 \%$ & $41 \%$ & $55 \%$ & $78 \%$ & $89 \%$ & $67 \%$ \\
\hline $5-10 a$ & $19 \%$ & $17 \%$ & $22 \%$ & $18 \%$ & $22 \%$ & $30 \%$ & $22 \%$ & 0 & 0 \\
\hline $10 \mathrm{a}$ & $25 \%$ & $25 \%$ & $8 \%$ & $33 \%$ & $33 \%$ & $11 \%$ & 0 & 0 & 0 \\
\hline
\end{tabular}

Colunas 2 e 6 - todos os profssionais UTIN

Colunas 3 e 7 - Profissionais NT

Colunas 4 e 8 - Profissionais NS

Tabela 2. Contagem de freqüência no checklist pertencente à Avaliação do Comportamento do Bebê Pré-termo adaptado (ACBP) (ALS et al., 1982)

\begin{tabular}{|c|c|c|c|c|c|c|c|}
\hline 1 & 2 & 3 & 4 & 5 & 6 & 7 & 8 \\
\hline Sinais de Retraimento & $\%$ & $\%$ & $\%$ & Sinais de Aproximação & $\%$ & $\%$ & $\%$ \\
\hline Regurgitar & 93 & 92 & 100 & Extensão de língua & 47 & 39 & 78 \\
\hline Ter náuseas & 80 & 78 & 89 & Mão à face & 87 & 86 & 89 \\
\hline Soluçar & 70 & 69 & 67 & Emissão de sons & 67 & 67 & 67 \\
\hline Movimento peristáltico & 22 & 25 & 11 & Mãos juntas tocando-se & 44 & 42 & 56 \\
\hline Caretas, retração de língua & 71 & 64 & 100 & Pés juntos tocando-se & 42 & 36 & 67 \\
\hline Arqueamento do tronco & 53 & 47 & 78 & Entrelaçar os dedos & 24 & 17 & 56 \\
\hline Dedos espalhados & 40 & 36 & 56 & Aconchegar-se & 73 & 69 & 89 \\
\hline Asa de avião & 60 & 58 & 67 & Movimentos corporais & 64 & 61 & 78 \\
\hline Saudação & 49 & 44 & 67 & Mão à boca & 62 & 56 & 89 \\
\hline Sentado no ar & 13 & 6 & 44 & Movimentos de preensão & 38 & 22 & 100 \\
\hline Espirrar & 53 & 50 & 67 & Procura de anteparo para os pés ou pernas & 27 & 22 & 44 \\
\hline Bocejar & 80 & 75 & 100 & Reflexo de procura & 62 & 56 & 89 \\
\hline Suspirar & 44 & 42 & 56 & Sugar & 84 & 81 & 100 \\
\hline Tossir & 58 & 50 & 89 & Segurar a mão do examinador & 69 & 61 & 100 \\
\hline Desviar & 18 & 11 & 44 & Fazer "OOH" com a boca (arredondando os lábios) & 36 & 28 & 67 \\
\hline \multirow[t]{2}{*}{ Franzir a testa } & 93 & 92 & 100 & Fixar-se visualmente ou auditivamente & 31 & 22 & 67 \\
\hline & & & & $\begin{array}{l}\text { Abocanhar: movimentos de abertura e fechamento } \\
\text { de boca }\end{array}$ & 80 & 78 & 89 \\
\hline
\end{tabular}

Ao analisar a Tabela 2, do total de 16 sinais de retraimento e 17 sinais de aproximação possíveis de assinalar, observou-se que sete sinais de retraimento (regurgitar, ter náuseas, caretas, arqueamento de tronco, bocejar, tossir, franzir a testa) foram listados por mais de
$70 \%$ dos profissionais do NS, enquanto que no NT apenas quatro sinais (regurgitar, ter náuseas, bocejar e franzir a testa) foram listados por mais de $70 \%$ destes. Nos sinais de aproximação mais de 70\% dos profissionais de NS listaram 10 sinais (extensão de língua, mão à face, aconchegar- 
se, movimentos corporais, mão à boca, movimentos de preensão, reflexo de procura, sugar, segurar a mão do examinador, abocanhar) e no NT foram listados três destes sinais (mão à face, sugar e abocanhar) por mais de $70 \%$ dos profissionais. Assim, nota-se que a equipe de NS observa três sinais de retraimento e sete sinais de aproximação a mais que o NT.

Os Gráficos A, B, e C apresentam os sinais de retraimentos e aproximação assinalados por mais de $70 \%$ dos profissionais; de $50 \%$ a $70 \%$ destes; e abaixo de $50 \%$. Ao comparar o gráfico do NS com o NT, observa-se uma inversão destes. No NT a maior parte dos sinais tanto de retraimento, quanto de aproximação são reconhecidos por poucos profissionais $(<50 \%)$, já no NS a maior parte destes sinais são reconhecidos pela maioria dos profissionais (> 70\%). Portando, nota-se que os profissionais de NS reconhecem mais sinais neurocomportamentais que os profissionais do NT.

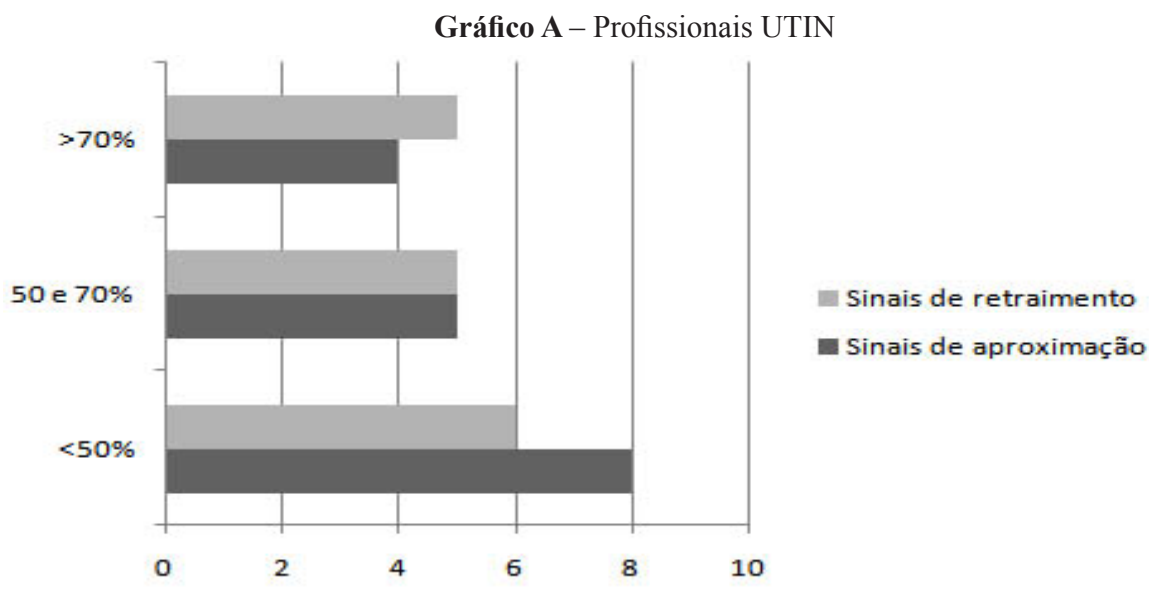

Gráfico B -Nível Técnico

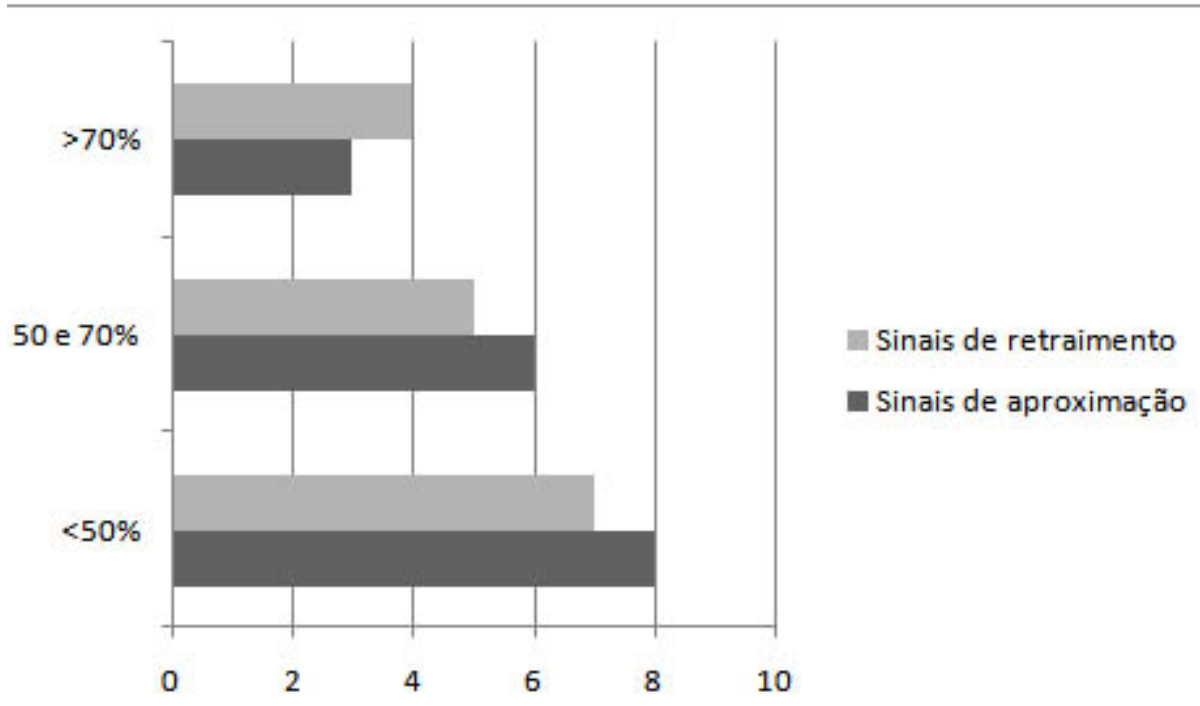

Dos profissionais que responderam ao checklist $36 \%$ (16 profissionais) responderam à parte qualitativa, sendo que três responderam à primeira pergunta; quatro à segunda; e 15 à terceira. A partir da análise de conteúdo destas respostas foram criadas quatro categorias temáti- cas: 1) Experiência influenciada pela extensiva jornada de trabalho; 2) Experiência e dificuldades relacionadas ao reconhecimento dos sinais; 3) Experiência através da observação ativa; e 4) Adquirindo experiência através de capacitações. 


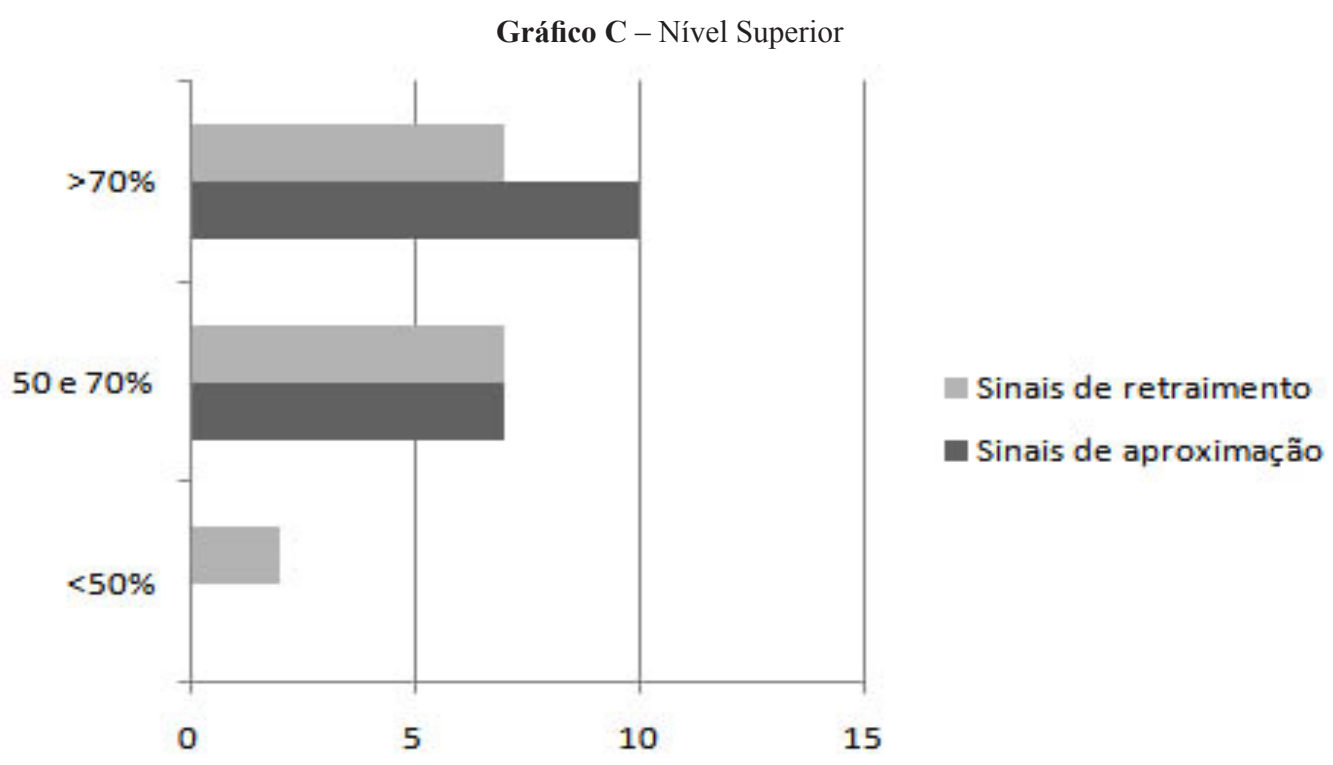

\section{DISCUSSÃO}

Nos resultados apresentados na Tabela 1 e nos Gráficos B e C notou-se que os sinais de aproximação possuem maior freqüência no NS, ou seja, que maior quantidade de profissionais desta categoria identifica os sinais neurocomportamentais. Isto nos leva a refletir sobre as condutas de assistência entre as categorias profissionais. Uma consideração importante para ser pensada é se existe diferença de assistência conforme a formação profissional: seria a intervenção da equipe de NS mais humanizada?; seriam as condições de trabalho da equipe do NT mais desgastantes, com tempo muito pequeno para a intervenção o que dificulta a identificação, envolvendo assim, fatores que constituem obstáculos para a oferta da assistência humanizada?; ou ainda, seria a linguagem utilizada pelo checklist muito técnica dificultando o entendimento do conteúdo?

Murdoch e Danford (1993) apud Ramos (2004), verificaram que as manipulações nos RNPT chegaram a ocorrer em média a cada 5-20 minutos, sendo os técnicos e auxiliares de enfermagem os principais responsáveis por tais intervenções, seguidos pelos enfermeiros, pediatras e finalmente pelos pais. Além da equipe técnica ser a maior agente de procedimentos diretos com os bebês em UTIN, Souza e Ferreira (2010), apontam que muitas UTIN's funcionam com equipes reduzidas desses profissionais o que interfere de forma direta na qualidade da produção de cuidados, provocando uma sobrecarga de trabalho. Deve-se também considerar a disponibilidade de acesso a estas informações nos diferentes níveis durante sua formação. Estes fatores podem representar obstáculos para a observação ativa dos sinais neurocomportamentais e, conseqüentemente, para a oferta do cuidado humanizado ao bebê prematuro.

Nos resultados dos sinais de aproximação obtiveramse quatro destes sinais identificados por mais de $70 \%$ dos profissionais de NT e 10 sinais por mais de $70 \%$ dos profissionais do NS. As intervenções junto aos sinais de aproximação apresentados pelo bebê visam sua organização e refletem sua habilidade em estabelecer um nível de funcionamento integrado entre os sistemas fisiológicos e comportamentais (BRASIL, 2009; KUDO et al., 1997). Estas intervenções, além de afetar positivamente a sobrevivência do bebê, permeia todas suas interações no meio e, conseqüentemente, promove seu desenvolvimento neurológico (BRASIL, 2009; TAMEZ, 2009).

Em relação aos sinais de retraimento, mais de $70 \%$ dos profissionais do NT listaram quatro destes sinais, em contrapartida, mais de $70 \%$ dos profissionais de NS listaram 07 sinais de retraimento. Observou-se que tanto no NT, quanto no NS os sinais de retraimento de maior frequência estão relacionados aos subsistemas motor e autonômico. Brasil (2009) e Tamez (2009), apontam que no bebê prematuro a energia disponível para os subsistemas está distribuída de forma diferenciada do bebê a termo. Há uma grande demanda para o subsistema autônomo seguido pelo motor para o funcionamento adequado destes restando pouca energia para os demais. O bebê prematuro possui 
baixo limiar para responder a estímulos vindos do meio e ao receber um estímulo inadequado responde principalmente com os sinais de retraimento dos subsistemas autonômico e motor. Assim, o bebê utiliza grande parte da energia com os sinais de retraimento em resposta ao estímulo, sendo que esta deveria ser utilizada para o funcionamento adequado desses subsistemas. Portanto haverá uma alteração da homeostase do bebê, que poderá ser expressa em taquicardia, apnéia, hipotonia, entre outros (BRASIL, 2009; TAMEZ, 2009).

A alta freqüência dos sinais de retraimento apresentados neste estudo induz a reflexão sobre as formas de assistência dentro das UTIN's. Segundo Brasil (2009), estes sinais indicam a necessidade de pausa dos procedimentos, ou que sejam feitas manobras como contenção facilitada ou enrolamento para a organização deste bebê, ou até mesmo a suspensão temporária do procedimento. Atitudes como estas indicam uma atuação profissional mais humanizada, na qual os profissionais além de utilizar adequadamente os aparatos tecnológicos, realizarão uma intervenção que considera as necessidades reais do bebê e que respeitam sua maturidade neurológica.

Esta proposta de atenção junto aos sinais neurocomportamentais de cada bebê vai de encontro ao proposto pela PNH dentro de seus princípios norteadores sobre o acolhimento, em que os profissionais devem realizar a observação e escuta atenta, sendo capaz de perceber as diversas demandas. O reconhecimento, por parte da equipe de saúde das condições nas quais se encontram o corpo e a subjetividade do paciente é o passo inicial para a incorporação de uma nova forma de compreensão do acolhimento e da assistência (ALVES et al., 2009).

Muitos dos dados quantitativos se justificam nas categorias criadas na análise dos dados qualitativos. $\mathrm{Na}$ categoria "Experiência profissional influenciada pela extensiva jornada de trabalho", foi verificado que a jornada de trabalho excessiva e as demandas de atuações pontuais e certeiras, em prol da manutenção da vida dos bebês, exigem esforços extras dos profissionais e podem influenciar a sua percepção dos sinais neurocomportamentais. Tal demanda pode ser vista no relato de um dos profissionais:

“...porém, temos pouco tempo para observar esses sinais, devido aos cuidados intensos que nos deixam sem tempo para esses comportamentos e sinais do bebê" $\left(\mathrm{S}_{3}-\right.$ técnico de enfermagem).

A literatura pontua constantemente a sobrecarga de trabalho sobre médicos e enfermeiros que atuam em UTIN's (FOGAÇA et al., 2008, 2009; OLIVEIRA et al., 2006). O ambiente fechado e estressante com barulho constante dos aparelhos, a necessidade de alta qualidade técnica dos procedimentos, a atuação com o limiar entre a vida e a morte e o lidar com o sofrimento do paciente e da família são fatores estressantes e que influenciam no desgaste profissional (COUTRIN et al., 2003). Segundo Fogaça et al. (2008) vários estudos apontam as repercussões desses fatores na saúde física, mental e social do trabalhador da UTIN como: alterações psicológicas; insatisfação com o sono; dependência de medicamentos; dificuldades de relacionamento com a equipe, pacientes e familiares; entre outros. O profissional submetido a esta rotina pode se tornar vulnerável, prestando uma assistência mecanizada e tecnicista, não reflexiva, esquecendo de humanizar o cuidado (COLLET; ROZENDO, 2003). Assim, para oferecer o cuidado humanizado se faz necessária a humanização das condições de trabalho dos profissionais, sendo este ponto discutido desde o processo de construção do Sistema Único de Saúde (SUS). Souza; Mendes, 2009 apontam que os trabalhadores precisam de melhores condições de trabalho, de formação continuada e até mesmo de apoio psicológico, para lidar satisfatoriamente com a intensidade do impacto que o enfrentamento cotidiano da doença e do sofrimento impõe. Porém, deve-se também refletir acerca da atribuição ao outro a responsabilidade pelas falhas na humanização, e nesse momento há o risco do profissional se isentar da transformação e colocar-se repetindo modos que critica, gerando falas como: "É assim, sempre foi assim...", "Se os gestores não fazem a parte deles, não há o que fazer" (PEDROSO; VIEIRA, 2009).

$\mathrm{Na}$ categoria "Experiência e dificuldades relacionadas ao reconhecimento dos sinais", os profissionais pontuaram que ainda há incerteza sobre os sinais, pois eles podem ser muito sutis o que pode levar ao não reconhecimento e possível desvalorização de sua importância para o desenvolvimento do bebê. Tal fato pode ser vista na fala:

“... esses sinais são leves e quase sutis, que temos que estar bem atentos para identificá-los, o que dificulta muito o reconhecimento." $\left(\mathrm{S}_{5}\right.$ - auxiliar de enfermagem)

Santos (2007), aponta que a falta de respostas específicas a estímulos que geram desconforto, faz com que a observação superficial do comportamento de um recém nascido não permita perceber nuances discretas de suas interações com o meio. Devido às peculiaridades das respostas dos RNPT aos estímulos recebidos, estes foram considerados desorganizados e sem uma estrutura que possibilitasse avaliações na esfera neurocomportamental de sua maturação neurológica. Somente no início do século XX estes bebês passaram a ser vistos como alguém de quem se poderia extrair sinais que apontassem para a existência, 
já em fase precoce, de habilidades funcionais (LESTER; TRONICK, 2004).

$\mathrm{Na}$ categoria "Experiência através da observação ativa", os profissionais pontuaram que quanto maior o tempo de atuação junto aos bebês prematuros, mais experientes se tornam na identificação dos sinais neurocomportamentais. Este fato é observado nos relatos:

“...aprendi a identificar os sinais com o tempo de trabalho no setor." ( $\mathrm{S}_{5}$ - auxiliar de enfermagem)

"Todo corpo fala e com prematuros não é diferente, se sabemos observá-los, sabemos até mesmos os sinais quando tem fome; observação é uma técnica que tem que ser feita diariamente." ( $\mathrm{S}_{7}$ - auxiliar de enfermagem)

Estudos apontam que além da prática profissional é essencial a constante atualização de conhecimentos teóricos evitando assim a atuação apenas nos conhecimentos adquiridos nas práticas diárias. Souza et al. (2006), afirmam que até 1940 o foco da enfermagem centrava-se nas tarefas e procedimentos, sem levar em consideração a construção intelectual, e este fato ainda se reproduz, principalmente, aos técnicos e auxiliares de enfermagem, estando estes mais voltados à técnica que a atualização de conhecimentos teóricos. Bernardo et al. (2005) em uma revisão de literatura avaliaram a relação entre a qualidade de atenção em saúde e o tempo de prática médica. No total, 32 de 62 estudos $(52 \%)$ demonstraram que a performance diminuiu à medida que a experiência aumentou; e apenas (2\%) referiu uma associação positiva, em que a performance cresce à medida que a experiência cresce. Portanto, esta revisão de literatura em concordância com o citado por Souza et al. (2009) indicam que apesar da experiência clínica, os profissionais necessitam de atualizações de conteúdo visando melhor qualidade na atenção à saúde e promovendo assim uma prática clínica baseada em evidências científicas.

Na última categoria nomeada "Adquirindo experiência através de capacitações", os pesquisados apontam a necessidade do aperfeiçoamento e transmissão de informação a todos os profissionais atuantes em UTIN sobre os sinais neurocomportamentais e sua importância para o desenvolvimento saudável do bebê e para uma prática mais humanizada.

"Percebo alguns profissionais da equipe têm pouco conhecimento sobre tais comportamentos tão importantes para o desenvolvimento do bebê. Penso que deveria ser passado à toda a equipe maiores informações e conhecimentos à esse respeito (sinais neurocomportamentais)" $\left(\mathrm{S}_{8}-\right.$ psicóloga $)$

Als (2009) pontua que os profissionais da UTIN devem ser orientados e recomendados a realizar uma observação detalhada do bebê durante os cuidados diários, a fim de minimizar o estresse da criança e otimizar o seu desenvolvimento. Assim, destaca-se a importância da capacitação dos profissionais, sendo que esta pode ser realizada por meio da educação continuada em saúde. Segundo Fonseca et al. (2004) esta educação tem o objetivo de promover transformações na compreensão da saúde, relacionando à qualidade e compromisso com a vida, gerando novas atitudes na assistência. Esta pode ser realizada através de dinâmicas grupais, materiais educativos e cursos teóricos e práticos, entre outros. Ao adquirir estes conhecimentos, os profissionais poderão transmití-los a novos profissionais que irão compor a equipe da UTIN e aos pais das crianças na UTIN (BRASIL, 2009).

\section{CONCLUSÕES}

Existem diversas ações que efetivamente podem interferir na assistência humanizada como: a intervenção junto aos sinais neurocomportamentais do RNPT na UTIN; a organização do ambiente físico; a organização da rotina dos procedimentos; o melhor manejo nas intervenções diretos com o pré-termo; o maior tempo de visita dos pais à UTIN; oferecer apoio aos pais em relação ao reconhecimento da linguagem e dos cuidados com o bebê; e a humanização das condições de trabalho dos profissionais que atuam na UTIN.

Os profissionais atuantes na busca da humanização do cuidado junto ao RNPT nas UTIN's deverão promover todos estes aspectos. Ao realizar estas ações, o profissional estará prevenindo possíveis seqüelas que reduziriam o desempenho ocupacional da criança, e promovendo o seu desenvolvimento saudável. Além disso, estes profissionais poderão promover a qualidade de vida de todos envolvidos no acolhimento ao RNPT de forma mais humanizada.

A capacidade do profissional observar e intervir junto aos sinais neurocomportamentais é um dos passos para a humanização do atendimento. Destaca-se que essas ações não dependem de tecnologia e grande disponibilidade de recursos financeiros, porém esta prática é influenciada por diversos fatores como: sobrecarga de trabalho; sutileza dos sinais; a observação ativa na prática diária; e a necessidade de capacitações para tal. Este último fator influencia todos os anteriores, pois, antes de tudo, para conseguir identificar e intervir junto a estes sinais, é necessário a capacitação teórico-prática e a sensibilização dos profissionais para que estes adquiram e articulem esses conhecimentos em suas práticas diárias facilitando assim o manejo com o RNPT. Uma das bases teóricas para esta capacitação acerca dos 
sinais neurocomportamentais é a teoria Síncrono Ativa do Desenvolvimento (ALS, 1986), sendo esta a teoria mais aceita atualmente, em que utiliza estes sinais como um ponto de partida e de modulação de estímulos.
Em relação à UTIN pesquisada, observa-se a necessidade de maior sensibilização dos profissionais desta UTIN quanto a importância de maiores ações em prol da humanização do atendimento.

ALMOHALLA, L., GUERRA, R. M. R. Identification of the preterm neurobehavioral signs by the staff in Neonatal Intensive Care Unit (NICU). Rev. Ter. Ocup. Univ. São Paulo, v. 22, n. 2, p. 117-126, maio/ago. 2011.

\begin{abstract}
Introduction: In response to external stimuli intrinsic to the neonatal intensive care unit (NICU), the preterm infant exhibits neurobehavioral signs of avoidance cues or approach behavior. These signs offer tips to the professionals about their interventions. The correct modulation of stimuli leads to a better biological, social and emotional development of preterm infant and the care professionals must be prepared to perceive and evaluate these signs. Objective: Investigate the level of identification by NICU professionals, of the neurobehavioral signs of avoidance cues and approach behavior, exhibited by preterm infants and analyze the factors that influence the acquisition of the knowledge about these signs. Methodological procedures: The data were collected through the applications of The Checklist of The Assessment of the Preterm Infants' Behavior (APIB) (ALS \& cols., 1982) to the NICU professionals' and through the analysis of the responses to the open questions included in the instrument. The quantitative data were analyzed by frequency count and the qualitative data by content analysis. Results: Forty-five professionals answered the checklist, $80 \%$ from the nursery assistant level and $20 \%$ from the graduated level. From the 16 signs of avoidance cues and from the 17 signs of approach behaviors present in the APIB Checklist, the graduate level professionals observed tree signs of avoidance cues and seven signs of approach behavior more than the nursery assistant professionals observed. Through the qualitative analysis, it was verified that the correct identifications of the preterm infants' signs by the professionals is influenced by factors such as a heavy load of work, subtlety of the signs showed by babies, professional experience and the need of continued education.
\end{abstract}

KEY WORDS: Humanization of assistance; Neuroberavioral manifestations; Infant,premature; Health personnel.

\section{REFERÊNCIAS}

ALS, H. A synactive model of neonatal behavioral organization: framework for the assessment of neurobehavioral development in the premature infant and for support of infants and parents in the neonatal intensive care environment. Phys. Occup. Ther. Pediatr., v. 6, n. $3 / 4$, p. $3-53,1986$.

ALS, H. Newborn Individualized Developmental Care and Assessment Program (NIDCAP): new frontier for neonatal and perinatal medicine. J. Neonatal-Perinatal Med., Washington, v. 2, p. 135-147, 2009.

ALS et al. Towards a research instrument for assessment of preterm infant behavior (APIB) and the manual for assessment of preterm infant behavior. In: FRITZGERALD, H. E.; LESTER, B. M.; YOGMAN, M. W. Theory and research in behavior pediatrics. New York: Plenum Press, 1982.

ALVES, C. A.; DESLANDES, S .F.; MITRE, R. M. A. Challenges of humanization in the context of pediatric nursing care of medium and high complexity. Interface Comun. Saúde Educ., Botucatu, v. 13, supl. 1, p. 581-94, 2009.

ANDREA, M. O. RODOLFO, B. L. Terapia ocupacional neonatal, una propuesta para la acción. Rev. Chil. Ter. Ocup., Santiago, n. 6, p. 23-32, 2006.

BARDIN, L. Análise de conteúdo. Lisboa: Edições 70, 1979.

BERNARDO, W. M.; JATENE, F. B.; NOBRE, M. R. C. Experiência clínica, educação médica continuada e qualidade da atenção em saúde. Rev. Assoc. Med. Bras., São Paulo, v. 51, n. 2, p. 63-64, 2005 .

BRASIL. Ministério da Saúde. Secretaria de Atenção à Saúde. Área de Saúde da Criança. Atenção humanizada ao recém-nascido de baixo peso: Método Canguru/ Ministério da Saúde, Secretaria de Atenção à Saúde, Área Técnica da Saúde da Criança. - Brasília: Ministério da Saúde, 2009. Disponível em: http://www.aleitamento. com.br/upload/arquivos/arquivo1_2039.pdf. Acesso: 14 mar. 2010 .

BRASIL. Ministério da Saúde. Secretaria de Atenção à Saúde. Núcleo Técnico da Política Nacional de Humanização. HumanizaSUS: Documento base para gestores e trabalhadores do SUS / Ministério da Saúde, Secretaria de Atenção à Saúde, Núcleo Técnico da Política Nacional de Humanização- 4. ed. - Brasília : Editora do 
Ministério da Saúde, 2008. Disponível em: bvsms.saude.gov.br/.../ humanizasus_documento_gestores_trabalhadores_sus.pdf. Acesso em: 22 abr. 2010 .

BÜHLER, K. E. C. B. Desenvolvimento cognitivo e de linguagem expressiva em bebês pré-termo muito baixo peso em seus estágios iniciais. 2008. 201 f. Tese (Doutorado em Ciências da Saúde) - Departamento de Fisioterapia, Fonoaudiologia e Terapia Ocupacional. Faculdade de Medicina da Universidade de São Paulo, São Paulo, 2008.

CARVALHO, W. B.; PEDREIRA, M. L. G.; AGUIAR, M. A. L. Nível de ruídos em uma unidade de cuidados intensivos pediátricos. J. Pediatr., Rio de Janeiro, v. 81, n. 6, p. 495-498, 2005.

COLLET, N.; ROZENDO, C. A. Humanização e trabalho na enfermagem. Rev. Bras. Enferm., Brasília, v. 56, n. 2, p. 189-192, 2003.

COSTA, H. P. F.; MARBA, S. T. O recém-nascido de muito baixo peso. São Paulo: Atheneu, 2006.

COUTRIN, R. M. G. S.; FREUA, P. R; GUIMARÃES, C. M. Estresse em enfermagem: uma análise do conhecimento produzido na literatura brasileira no período de 1982 a 2001. Texto \& Contexto Enferm., Florianópolis, v. 12, n. 4, p. 486-494, 2003.

FOGAÇA, M. C.; CARVALHO, W. B.; CÍTERO, V.A.; NOGUEIRA MARTINS, L. A. Estresse ocupacional e suas repercussões na qualidade de vida de médicos e enfermeiros intensivistas pediátricos e neonatais. Rev. Bras. Ter. Intens., Rio de Janeiro, v. 21, n. 3, p. 299-305, 2009.

FOGAÇA, M. C.; CARVALHO, W. B.; CÍTERO, V. A.; NOGUEIRA MARTINS, L. A. Fatores que tornam estressante o trabalho de médicos e enfermeiros em terapia intensiva pediátrica e neonatal: estudo de revisão bibliográfica. Rev. Bras. Ter. Intens., Rio de Janeiro, v. 20, n. 3, p. 261-266, 2008.

FONSECA, L. M. M.; SCOCHI, C. G. S.; ROCHA, S. M. M.; LEITE, A. M. Cartilha educativa para orientação materna sobre os cuidados com o bebê prematuro. Rev. Latino-am. Enferm., Ribeirão Preto, v. 12, n. 1, p. 65-75, 2004.

GABRIEL, P. S. Z. Avaliação Neurocomportamental de bebês prematuros avaliados na fase de 32 a 37 semanas. 2008. 143 f. Dissertação (Mestrado em Ciências, Área: Psicologia) Departamento de Psicologia e Educação. Faculdade de Filosofia, Ciências e Letras de Ribeirão Preto/USP, Ribeirão Preto, 2008

KUDO, A. M.; et al. Fisioterapia, fonoaudiologia e terapia ocupacional em pediatria. 2a. ed. São Paulo: Sarvier, 1997.

LAMY,Z. C. P.; GOMES, R.; CARVALHO, M. A percepção de pais sobre a internação de seus filhos em unidade de terapia intensiva neonatal. J. Pediatr., Rio de Janeiro, v. 73, v. 5, p. 293-297, 1997.

LESTER, B. M.; TRONICK, E. Z. History and description of the neonatal intensive care unit network neurobehavioral scale.
Pediatrics, v. 113, p. 634-40, 2004.

MARTINS, J. J.; FARIA, E. M. A reorganização do trabalho da enfermagem em UTI. Texto \& Contexto Enferm., Florianópolis, v. 9, n. 2, p. 388-401, 2000.

MONTEIRO, R. C. S. Neonatalogia. In: CAVALCANTI, A.; GALVÃO, C. Terapia oOcupacional: fundamentação \& prática. Rio de Janeiro: Guanabara Koogan, 2007.

OLIVEIRA, B. R. G.; LOPES T. A.; VIERA C. S.; COLLET N. $O$ processo de trabalho da equipe de enfermagem na uti neonatal e o cuidar humanizado. Texto \& Contexto Enferm., Florianópolis, v. 15, n. esp., p. 105-113, 2006.

PEDROSO, R. T.; VIEIRA, M. E. M. Humanização das práticas de saúde: transversalizar em defesa da vida. Interface Comun. Saúde Educ., Botucatu, v. 13, supl. 1, p. 695-700, 2009.

PÊGO, J. MAIA, S. M. A importância do ambiente no desenvolvimento do recém-nascido pré-termo. Distúrb. Comun., São Paulo, v. 19, n. 1, p. 39-50, 2007.

RAMOS, M. NIDCAP ${ }^{\circledR}$ Uma realidade possível... Rev. Sinais Vitais, São Paulo, n. 54, p. 9-12, 2004.

REICHERT, A. P. S.; LINS, R. N. P., COLLET, N. Humanização do Cuidado da UTI Neonatal. Rev. Eletrônica Enferm., Goiânia, v. 9, n. 1, p. 200-213, 2007.

SANTOS, M.A.A. Perfil neurocomportamental de recém-nascidos pré-termo ao atingirem o termo. 2007. $131 \mathrm{f}$. Tese (Mestrado em Ciências da Saúde) - Pós-Graduação da Faculdade de Ciências Médicas da Santa Casa de São Paulo, São Paulo, 2007.

SCOCHI, C. G. S.; et al. Cuidado individualizado ao pequeno prematuro: o ambiente sensorial em unidade de terapia intensiva neonatal. Acta Paul. Enferm., São Paulo, v. 14, n. 1, p. 9-16, 2001.

SOUZA, et al. Formação do enfermeiro para o cuidado: reflexões da prática profissional. Rev. Bras. Enferm., Brasília, v. 59, n. 6, p.80-7, 2006.

SOUZA, K. M. O.; FERREIRA, S. D. Assistência humanizada em UTI neonatal: os sentidos e as limitações identificadas pelos profissionais de saúde. Ciênc. Saúde Coletiva, Rio de janeiro, V.15, n.2, p. 471-480, 2010

SOUZA, L. A. P.; MENDES, V. L. F. O conceito de humanização na Política Nacional de Humanização (PNH). Interface Comun. Saúde Educ., Botucau, v.13, supl.1, p.681-8, 2009.

THAMEZ, R. N. Intervenções no cuidado neuropsicomotor do prematuro: UTI neontatal. Rio de Janeiro: Guanabara Koogan, 2009.

TRONCHIN, D. M. R.; TSUNECHIRO, M. A. Prematuros de muito baixo peso: do nascimento ao primeiro ano de vida. Rev. Gaúch. Enferm., Porto Alegre, v. 28, n. 1, p. 79-88, 2007. 